\title{
Temperature effects in the blown Newtonian film
}

\author{
J. C. Bennett ${ }^{1} \quad$ J. J. Shepherd ${ }^{2} \quad$ W. F. Blyth ${ }^{3}$
}

(Received 31 July 2007; revised 20 November 2007)

\begin{abstract}
The film blowing process, by which thin polymer films are manufactured, is modelled by highly nonlinear differential equations. Typically, analysis of these using numerical techniques leads to instabilities. Our earlier work used an iteration process to construct the film bubble profile and some associated quantities for the simplest case of a Newtonian film incorporating an axial temperature variation. Now we extend this to an investigation of the effects of variations in temperature parameters on the film radius, film fluid velocity, film thickness and overall temperature profiles. This information is of considerable use to investigators examining the film blowing process.
\end{abstract}

\section{Contents}

1 Introduction

C216

See http://anziamj.austms.org.au/ojs/index.php/ANZIAMJ/article/view/354 for this article, (c) Austral. Mathematical Soc. 2007. Published December 20, 2007. ISSN 1446-8735 
2 Non-dimensional governing equations

2.1 The analytical approximation . . . . . . . . . . . C C219

3 Linearization and the iteration procedure

4 Results and discussion

$\mathrm{C} 222$

References

$\mathrm{C} 227$

\section{Introduction}

Film blowing is a well established industrial process employed in the manufacture of thin polymer film used in a variety of commercial and domestic applications.

A brief description of the process follows. Molten polymer is extruded vertically up through a thin horizontal annular die at a constant exit velocity. The resulting polymer tube is drawn upwards, while an applied internal pressure difference causes this tube to expand to an increased radius, forming a polymer bubble. Cooling by external air jets causes this bubble to solidify at the freezeline some distance above the extrusion point. The resulting cooled polymer tube is then wound onto rollers as a flat double layered film. Shepherd and Bennett [5] gave a schematic diagram of the apparatus involved. The mathematical modelling when the blown film is an isothermal Newtonian fluid is well established [4], while thermal effects in an analogous situation were introduced by Luo and Tanner [2].

In appropriate circumstances, the radial expansion above occurs in a relatively small part of the overall bubble region. This interior layer structure was exploited by Shepherd and Bennett [5] (and earlier by Shepherd, Connell and Tam [6]), where a singular perturbation constructed an analytic approximation to the radial film bubble profile for the simplest case of an isothermal 
Newtonian film. Subsequent work extended this to include a temperature variation along the film [1]; while a numerical process using the analytical approximation as initial iterate yielded an improved approximation to the bubble's radial and thermal profiles. This iterative process, based as it was on Maple's internal two point boundary value problem solver was computationally expensive. In this article we improve the iterative solution procedure by using a Galerkin finite element method. This numerical procedure is then applied to investigate the effects of film temperature variation on the radius, velocity and thickness of the film in the blowing process.

\section{Non-dimensional governing equations}

The bubble blowing process is assumed to display axial symmetry, with axis of symmetry the line normal to the plane of the annular die and through its centre. With $z$ taken as a dimensionless co-ordinate along this axis, where $z=0$ coincides with the plane of the exit die, the process is completely described by three dimensionless functions of $z$ : the film radius $r(z)$, film velocity $u(z)$ and film temperature $s(z)$. Consideration of the equilibrium of the forces acting on a film element and heat flow within that element then lead to the governing set of nonlinear differential equations

$$
\begin{aligned}
2 C^{2} r^{2}\left(f_{0}+B\left(r^{2}-1\right)\right) r^{\prime \prime}-6 C \eta r^{\prime} & \\
-r\left(f_{0}-B\left(3 r^{2}+1\right)\right)\left(1+C^{2}\left(r^{\prime}\right)^{2}\right) & =0, \\
2 C\left(2 r u^{\prime}+u r^{\prime}\right)-\frac{r u}{\eta}\left(f_{0}+B\left(r^{2}-1\right)\right)\left(1+C^{2}\left(r^{\prime}\right)^{2}\right) & =0, \\
s^{\prime}+H r \sqrt{1+C^{2}\left(r^{\prime}\right)^{2}}\left(s-s_{a}\right)+J r \sqrt{1+C^{2}\left(r^{\prime}\right)^{2}}\left(s^{4}-s_{a}^{4}\right) & =0,
\end{aligned}
$$

along with the boundary conditions,

$$
\begin{aligned}
& r(0)=1, \quad r^{\prime}(1)=0, \\
& u(0)=1, \quad s(0)=1 .
\end{aligned}
$$


Equations (1) and (2) result from stress balance on a film element in the meridional (longitudinally, along the bubble) and hoop (circumferentially, around the bubble) directions respectively. Derivations (in slightly different notation) are given by Pearson and Petrie [3, 4], with thermal effects included by Luo and Tanner [2]. The positive parameters $B, C$ and $f_{0}$ are dimensionless forms of the internal air pressure difference across the film, aspect ratio (radius of exit die to distance to freezeline) of the film bubble and pulling force at the die exit, respectively. The second of (4) reflects the fact that the freezeline occurs at $z=1$.

The properties of the film material enter into (1) and (2) through the factor $\eta$, a dimensionless Newtonian viscosity displaying an Arrhenius temperature variation, and defined by

$$
\eta(z)=\exp \left[w\left(\frac{1}{s(z)}-1\right)\right]
$$

In this, $w$ is a dimensionless form of the activation energy. Note that for the isothermal case, $\eta \equiv 1$ (or $w \equiv 0$ ).

Equation (3) describes heat flow in the film element, with the second term arising from Fourier law conduction and the third from radiation. The thermal parameters $H, J$, and $s_{a}$ are the dimensionless overall heat transfer coefficient, emissivity and ambient temperature respectively.

The non-dimensional bubble thickness $e(z)$ is defined through mass conservation by

$$
r(z) e(z) u(z)=1 .
$$

Equation (2) is integrated using $u(0)=1$ to obtain an integral expression for $u(z)$,

$$
u(z)=r^{-1 / 2}(z) \exp \left[\int_{0}^{z} \frac{\left(f_{0}+B\left(r^{2}(s)-1\right)\right)\left(1+C^{2}\left(r^{\prime}(s)\right)^{2}\right)}{4 C} d s\right] .
$$


Equation (7) is then rearranged to give

$$
e(z)=r^{-1 / 2}(z) \exp \left[-\int_{0}^{z} \frac{\left(f_{0}+B\left(r^{2}(s)-1\right)\right)\left(1+C^{2}\left(r^{\prime}(s)\right)^{2}\right)}{4 C} d s\right] .
$$

\subsection{The analytical approximation}

Shepherd and Bennett [5] used a combination of heuristic techniques and the method of matched asymptotic expansions was used to construct a closed form approximation for the bubble film radius in the case of the isothermal Newtonian film. Explicitly, this is

$$
r_{a}(z, C)=r_{1}(z, C)[H(z)-H(z-a)]+r_{2}(z, C)[H(z-a)-H(z-1)],
$$

where $H(z)$ is the Heaviside function, and

$$
\begin{aligned}
r_{1}(z, C)= & \phi(z, C)+(\phi(a, C)-\lambda) \operatorname{erf}\left(\frac{\sqrt{2 M}(a-z)}{2 C}\right) \\
& +\lambda-\phi(a, C),
\end{aligned}
$$

where

$$
\phi(z, C)=\left\{\left(1-\frac{3 B}{f_{0}-B}\right) \exp \left[\left(\frac{f_{0}-B}{3 C}\right) z\right]+\frac{3 B}{f_{0}-B}\right\}^{-1 / 2},
$$

while

$$
r_{2}(z, C)=\lambda+\frac{1}{18} \rho_{B U}^{3} \sigma \gamma\left(e^{\nu(z, C)}-e^{\nu(a, C)}\right)-\frac{\rho_{B U}}{6 C} \gamma(z-a),
$$

with

$$
\sigma=f_{0}+B\left(\rho_{B U}^{2}-1\right), \quad \gamma=f_{0}-B\left(3 \rho_{B U}^{2}+1\right),
$$


and

$$
\nu(z, C)=\frac{3(z-1)}{C \sigma \rho_{B U}^{2}} .
$$

Here, $\rho_{B U}$ the blow up ratio is the ratio of the bubble radius at the freezeline to the initial radius. The approximation (10) has the advantage of being explicit, and shows encouraging consistency when compared with numerical isothermal solutions [5]. It is useful as a starting point for an iterative scheme to construct numerical solutions to the model with temperature variation, as considered here.

\section{Linearization and the iteration procedure}

Equations (1) and (3) are coupled via the function $\eta(z)$, so that Equations (1) and (3) must be solved simultaneously. The procedure used here decouples Equations (1) and (3) with a solution being found by a linearization process leading to a series of 'simpler' differential equations, which are then solved numerically to iterate towards a solution of the full problem. This is similar to the approach of Bennett and Shepherd [1].

For given $s$, Equation (1) is written as

$$
N[r] \equiv C^{2} r^{\prime \prime}+f\left(r, r^{\prime}, s, C\right)=0
$$

where

$$
f\left(r, r^{\prime}, s, C\right)=\frac{\left(3 B r^{2}-f_{0}+B\right) r\left[1+C^{2}\left(r^{\prime}\right)^{2}\right]-6 C \eta r^{\prime}}{2 r^{2}\left[f_{0}+B\left(r^{2}-1\right)\right]}
$$

with $s(z)$ appearing in $(17)$ via $\eta(z)$. If $R(z)$ is some approximation to the solution of (16), by writing

$$
r(z)=R(z)+v(z)
$$


that equation is rearranged as

$$
C^{2} v^{\prime \prime}+f_{r^{\prime}} v^{\prime}+f_{r} v=-N[R]-Q\left(v, v^{\prime}, s, C\right),
$$

where $f_{r^{\prime}}$ and $f_{r}$ are the partial derivatives of $f$ with respect to the indicated variables evaluated at $\left(R, R^{\prime}, s, C\right)$ and

$$
Q\left(v, v^{\prime}, s, C\right)=f\left(R+v, R^{\prime}+v^{\prime}, s, C\right)-f\left(R, R^{\prime}, s, C\right)-v f_{r}-v^{\prime} f_{r^{\prime}},
$$

is quadratic in $v$.

The boundary conditions for $v(z)$ then become, from (18)

$$
v(0)=1-R(0), \quad v^{\prime}(1)=-R^{\prime}(1) .
$$

If, for given $s, R$ is a suitable approximation to $r, v$ will be appropriately small, and the system (19) and (21) may be solved iteratively as a linear nonhomogeneous two point boundary value problem.

The iteration procedure used to obtain a solution is similar to that described by Bennett and Shepherd [1]. Since the isothermal approximation (10) reflects the structure expected in the numerical solution (when $s=1), R(z)=r_{a}(z)$ is chosen here as a basis for an iteration process in (19). Thus, the nonlinear problem (19) and (21) is replaced by

$$
\begin{aligned}
& C^{2} v_{n}^{\prime \prime}+f_{r^{\prime}} v_{n}^{\prime}+f_{r} v_{n}=-N\left[r_{a}\right]-Q\left(v_{n-1}, v_{n-1}^{\prime}, s, C\right), \\
& v_{n}(0)=1-r_{a}(0), \quad v_{n}^{\prime}(1)=-r_{a}^{\prime}(0) .
\end{aligned}
$$

The two point boundary value problem (22) and (23) is solved using a Galerkin finite element procedure, with $v_{0}=0$ and an approximate temperature profile, $s_{0}$ is found by solving (3) subject to $s(0)=1$ with $r(z)=r_{a}(z)$, to obtain $v_{1} . v_{1}$ is then substituted into $Q\left(v, v^{\prime}, s, C\right)$ and the system (22) and (23) is solved again to yield a second update $v_{2}$ and so the process continues. The temperature contribution $s$ is updated after each iterate. The iteration process continues until a successive iterate absolute tolerance of $10^{-6}$ 


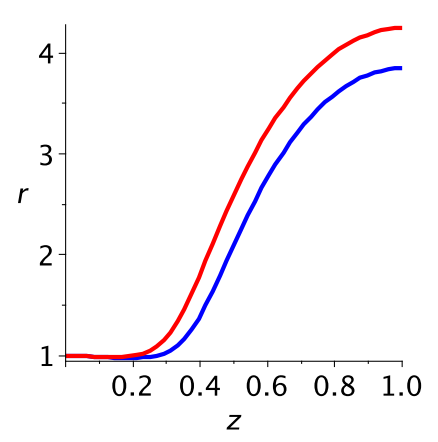

(a) Radius.

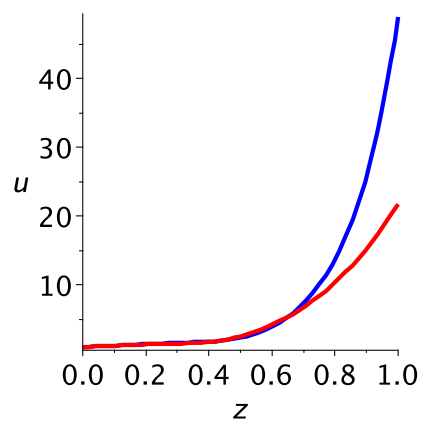

(b) Velocity.

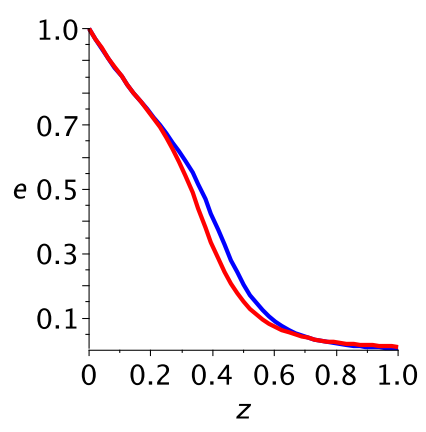

(c) Thickness.

Figure 1: Comparison between isothermal (blue) and thermal (red) radius, velocity, and thickness profiles. $B=0.21, C=0.15, f_{0}=0.969, H=0.01$, $J=0.03, w=7.448, s_{a}=0.1$.

is achieved upon which the residual is checked and the process terminates. Once a radial (and temperature) profile have been obtained, a velocity profile is found from (8) with $r(z)=r_{n}(z)$ and $s(z)=s_{n}(z)$, where $r_{n}(z)$ and $s_{n}(z)$ are the iterated solutions after $n$ iterates where tolerances have been met. A film thickness profile is found from (9) upon substitution of the radial and velocity solutions.

\section{Results and discussion}

The iterative process described in Section 3 based on the approximate isothermal profile (10) was applied for appropriate values of the bubble parameters, to obtain the bubble radius, film velocity and film thickness as functions of the axial variable $z$ on $0 \leq z \leq 1$.

Figure 1 compares these quantities in the isothermal $(\eta \equiv 1, w \equiv 0)$ and thermal cases. Evidently, the radius increases uniformly when a tem- 


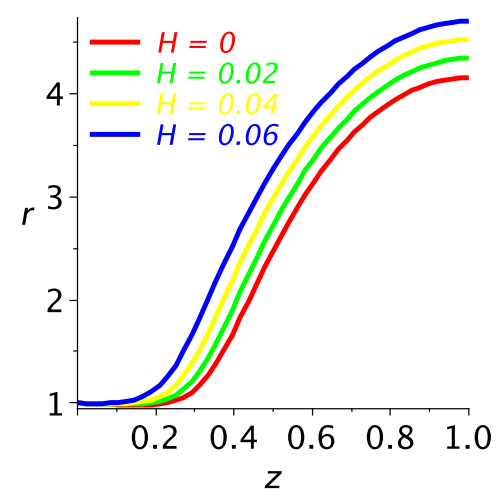

(a) Radius.

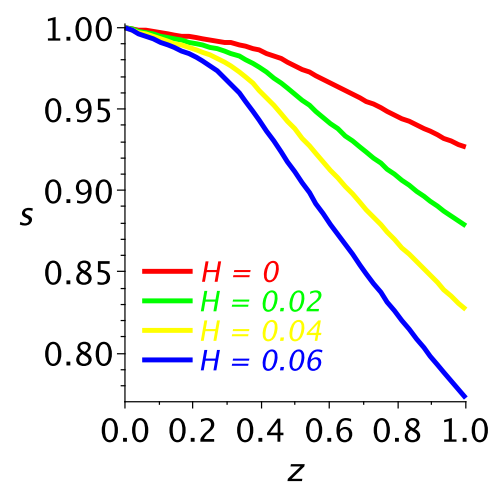

(c) Temperature.

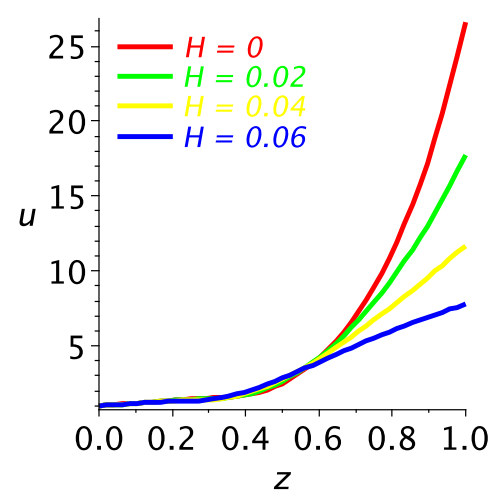

(b) Velocity.

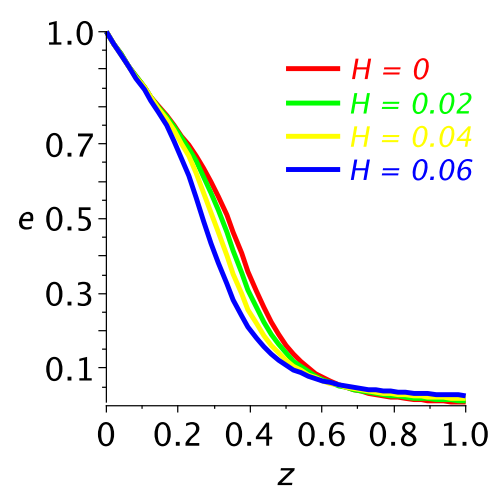

(d) Thickness.

Figure 2: Plot of radius, velocity, temperature and thickness profiles for varying values of $H$. 


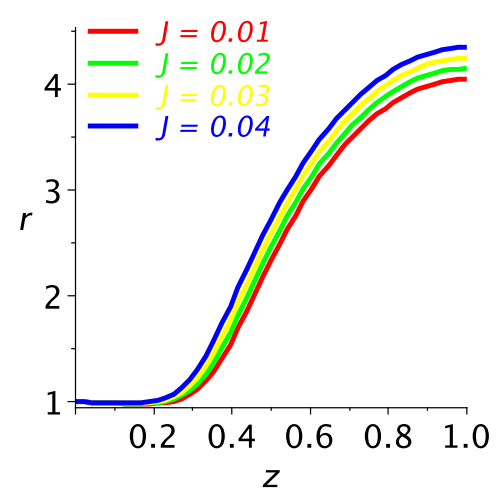

(a) Radius.

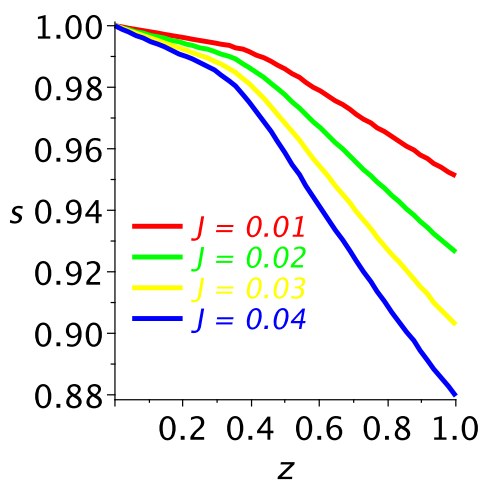

(c) Temperature.

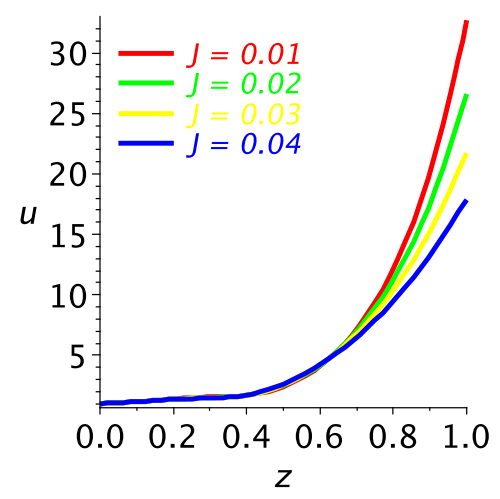

(b) Velocity.

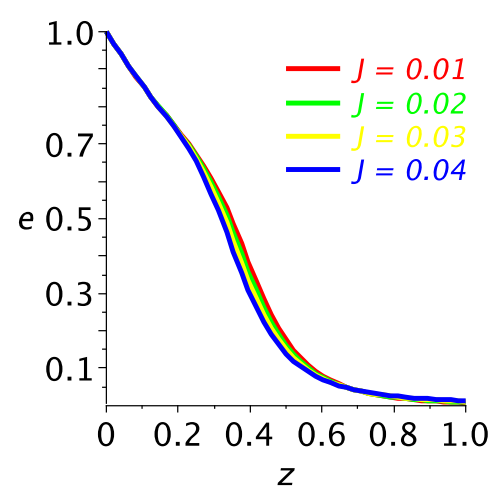

(d) Thickness.

Figure 3: Plot of radius, velocity, temperature and thickness profiles for varying values of $J$. 


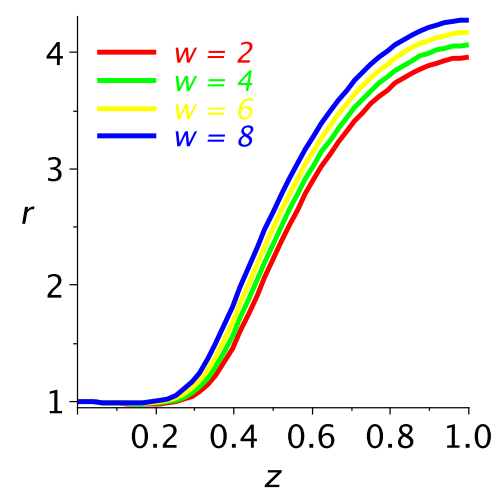

(a) Radius.

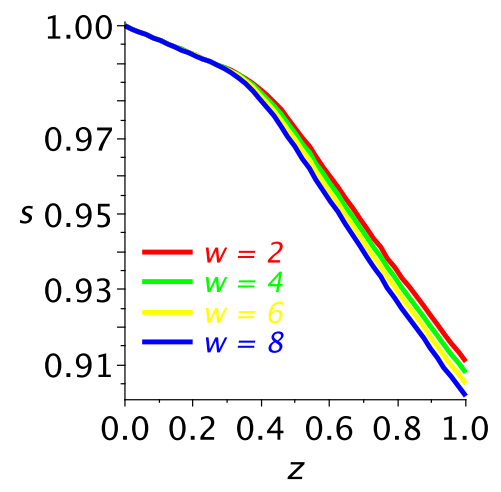

(c) Temperature.

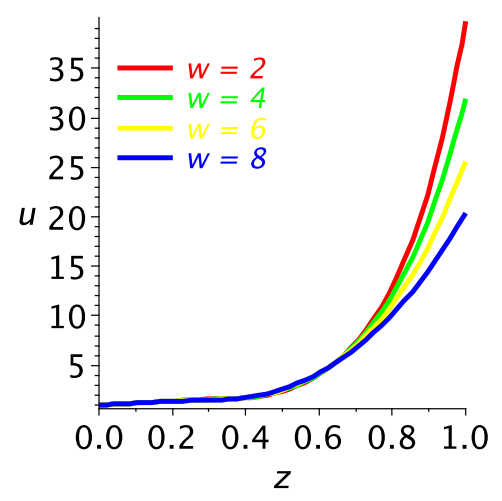

(b) Velocity.

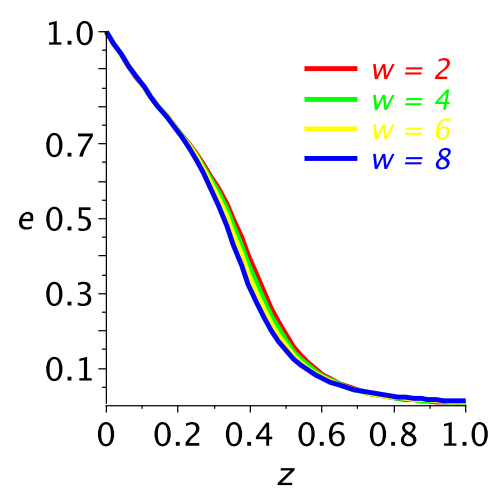

(d) Thickness.

Figure 4: Plot of radius, velocity, temperature and thickness profiles for varying values of $w$. 


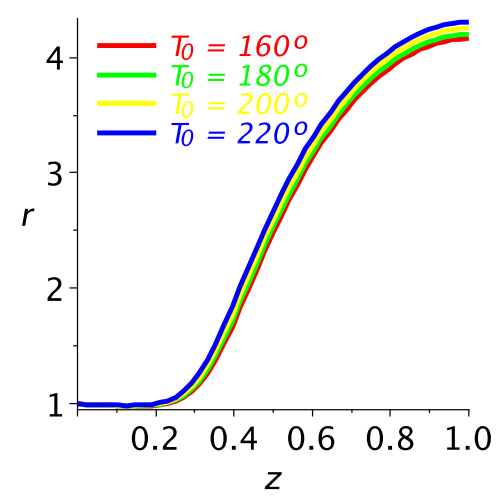

(a) Radius.

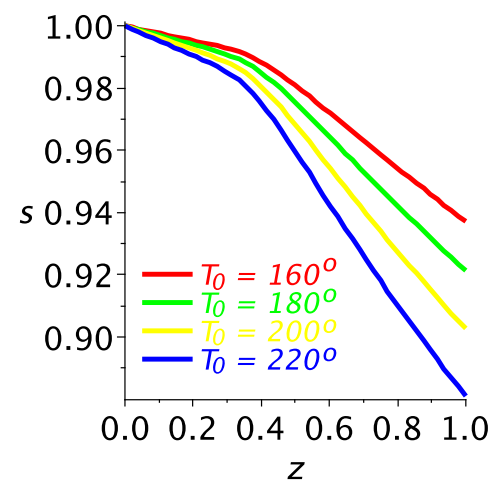

(c) Temperature.

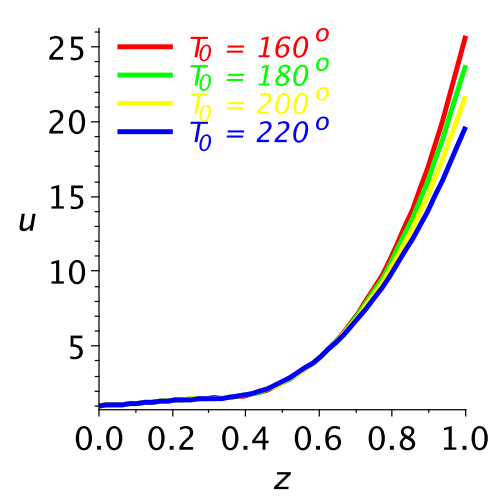

(b) Velocity.

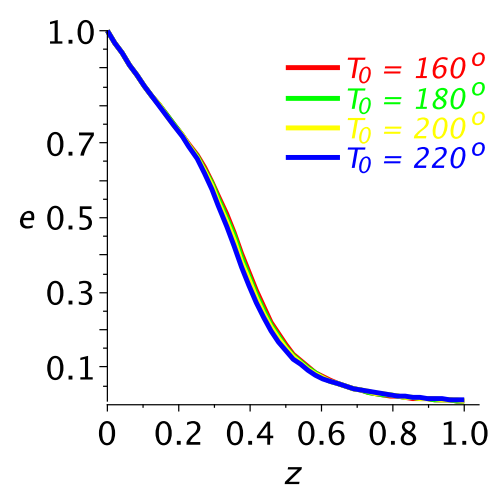

(d) Thickness.

Figure 5: Plot of radius, velocity, temperature and thickness profiles for varying values of $T_{0}$. 
perature profile is present, while the velocity profile changes dramatically, with temperature effects causing the freezeline velocity to reduce noticeably. Thermal effects are also evident in the thickness profile, with overall more rapid thickness reduction, but eventually a slightly larger thickness at the freezeline.

Figures 2, 3, 4 and 5 display variation of the radius, velocity, temperature and thickness profiles with the overall heat transfer coefficient, $H$, emissivity, $J$, activation energy, $w$ and extrusion temperature, $T_{0}$ respectively. In each case, increasing the relevant thermal parameter leads to a (generally) uniform increase in bubble radius (with corresponding increase in blow up ratio $\rho_{B U}$ ), and uniform decreases in the velocity, temperature and thickness profiles, with the most marked changes arising for variation of $H$ and $J$, the transfer coefficients in the temperature equation (3), and $T_{0}$, the film extrusion temperature (in the case of the bubble axial temperature profile). These reflect the general trends indicated in Figure 1. There are also subtle interchanges, particularly toward the freezeline $z=1$ in the film thickness, and mid-bubble in the film velocity.

\section{References}

[1] Bennett J. C. and Shepherd J. J., Analysis of the non-isothermal Newtonian model in the blown film process, ANZIAM Journal 47(E), 2006, pp. C325-C338.

http://anziamj . austms .org.au/V47EMAC2005/Bennett/ C217, C220, C221

[2] X.-L. Luo, R. I. Tanner, A computer study of film blowing, Polymer Engineering and Science, Volume 25, Number 10, 1985, pp. 620-629. C216, C218 
[3] J. R. A. Pearson, Mechanics of Polymer Processing, Elsevier Applied Science Publishers, 1985. C218

[4] J. R. A. Pearson and C. J. S. Petrie, The flow of a tubular film. Part 2. Interpretation of the model and discussion of solutions, Journal of Fluid Mechanics, Volume 42, Part 3, 1970, pp. 609-625. C216, C218

[5] Shepherd J. J. and Bennett J. C., Interior layer structure in the Newtonian blown film, ANZIAM Journal 46(E), 2005, pp. C839-C853. http://anziamj . austms.org.au/V46/CTAC2004/Shep/ C216, C219, $\mathrm{C} 220$

[6] J. J. Shepherd, H. J. Connell and D. C. H. Tam, An interior layer in a film-blowing problem, Proceedings of the Fifth Biennial Engineering Mathematics and Applications Conference (EMAC2002),

M. Pemberton, I. Turner, P. Jacobs (eds), The Institution of Engineers Australia, Brisbane, Queensland (2002), pp. 181-186. C216 


\section{Author addresses}

1. J. C. Bennett, School of Mathematical and Geospatial Sciences, Royal Melbourne Institute of Technology, Melbourne, Australia. mailto:E58144@ems.rmit.edu.au

2. J. J. Shepherd, School of Mathematical and Geospatial Sciences, Royal Melbourne Institute of Technology, Melbourne, Australia. mailto: jshep@rmit.edu.au

3. W. F. Blyth, School of Mathematical and Geospatial Sciences, Royal Melbourne Institute of Technology, Melbourne, Australia. mailto:bill.blyth@rmit.edu.au 